\title{
Phenotype Characteristics of Fellow Eyes in Patients With Early Onset of Neovascular Age-Related Macular Degeneration
}

\author{
Tina Schick, ${ }^{1,2}$ Lebriz Ersoy, ${ }^{1,2}$ Carel B. Hoyng, ${ }^{3}$ Bernd Kirchhof, ${ }^{2}$ and Sandra Liakopoulos ${ }^{2}$ \\ ${ }^{1}$ Cologne Image Reading Center, Department of Ophthalmology, University of Cologne, Cologne, Germany \\ ${ }^{2}$ Department of Ophthalmology, University of Cologne, Cologne, Germany \\ ${ }^{3}$ Department of Ophthalmology, Radboud University Nijmegen Medical Centre, Nijmegen, The Netherlands
}

Correspondence: Sandra Liakopoulos, Department of Ophthalmology, University of Cologne, Kerpener Strasse 62, 50924 Cologne, Germany;

Sandra.liakopoulos@uk-koeln.de.

Submitted: March 30, 2015

Accepted: October 6, 2015

Citation: Schick T, Ersoy L, Hoyng CB, Kirchhof B, Liakopoulos S. Phenotype characteristics of fellow eyes in patients with early onset of neovascular age-related macular degeneration. Invest Ophthalmol Vis Sci. 2015;56:7269-7273. DOI:10.1167/ iovs.15-16989
Purpose. To investigate phenotype characteristics of fellow eyes in patients with early onset of neovascular age-related macular degeneration (NVAMD).

Methods. Patients with new-onset unilateral NVAMD between 50 and 65 years $(n=57$, earlyonset choroidal neovascularization $[\mathrm{CNV}]$ group) or $>80$ years $(n=47$, late-onset $\mathrm{CNV}$ group) or with nonneovascular AMD ( $n=98$, no-CNV group) were included. Fellow eyes in both CNV groups and the eyes with the more severe AMD staging in the no-CNV group were used to evaluate number and size of macular drusen, extramacular drusen (EMD), pigmentary abnormalities, and retinal pigment epithelium (RPE) atrophy on color photographs and hyperreflective dots (HRD) and reticular pseudodrusen (RPD) on spectral-domain optical coherence tomography (SDOCT) scans. Regression analysis was used to compare groups.

RESULTS. Occurrence of $>20$ macular drusen was more frequent in the early-onset CNV group than the late-onset $\mathrm{CNV}$ group (odds ratio [OR] 2.93; $P=0.01$ ) or the no-CNV group (OR 2.17; $P=0.02$ ). Retinal pigment epithelium atrophy, RPD, and HRD appeared less frequently in the early-onset CNV group than in the late-onset CNV group (RPE atrophy: OR $0.11 ; P=$ 0.005 ; RPD: OR $0.04 ; P=9.38 \times 10^{-10}$, HRD: OR $0.30 ; P=0.004$ ) and no-CNV group (RPE atrophy: OR $0.12 ; P=0.005$; RPD: OR $0.40, P=0.03$, HRD: not significant). No differences were detected regarding presence of large drusen, pigmentary abnormalities, and EMD.

Concuusions. A large number of macular drusen in the fellow eye appeared to be characteristic for early onset of NVAMD, whereas RPE atrophy, HRD, and RPD were more frequently present in AMD patients $>80$ years. Prospective trials with patients converting to NVAMD are required to further analyze morphologic characteristics for early versus late development of advanced AMD.

Keywords: neovascular age-related macular degeneration, fellow eye, spectral-domain optical coherence tomography, reticular pseudodrusen
B efore anti-VEGF agents became available, age-related macular degeneration (AMD) was the leading cause of severe visual loss in people aged 50 years or older in the developed world. ${ }^{1}$ Multiple inherited and environmental factors play a role in the development and severity of this common disease. ${ }^{2}$ The incidence of typical AMD features, including drusen and pigmentary abnormalities, choroidal neovascularization (CNV), and geographic atrophy (GA), increases with age. ${ }^{3,4}$ Not only the phenotype but also the course of the disease varies strongly between individuals, with some patients developing CNV early and some patients never reaching this advanced stage of the disease. Once $\mathrm{CNV}$ develops, eyes are at high risk for a severe decrease in visual acuity. $^{5}$

The reason for those intraindividual differences is not yet fully understood. Smoking and genetic risk factors such as polymorphisms in $C F H$ and ARMS2 genes have been reported to be associated with a higher risk for neovascular AMD. ${ }^{6,7}$ Morphologic risk factors for the development of advanced AMD include large drusen, pigmentary abnormalities, and the involvement of the fellow eye. ${ }^{4,8}$ Recently, genetic risk factors for an early onset of CNV in AMD have been reported. ${ }^{9}$ However, morphologic parameters with a predictive value for an early onset of CNV in AMD still need to be determined.

Despite the large intraindividual variability, studies have reported a highly symmetrical appearance of both eyes of one patient. ${ }^{10}$ Additionally, various studies have proposed that patients with unilateral neovascular AMD have a substantial risk of developing CNV in their fellow eye. ${ }^{8,11-15}$ Therefore, in eyes with new-onset CNV, the morphologic appearance of the fellow eye may reveal risk factors for development of CNV.

Phenotyping in large epidemiologic AMD trials was mostly performed based on color fundus photographs. Since its first description in $1991,{ }^{16}$ optical coherence tomography (OCT) has been widely adopted in clinical routine and is increasingly used in clinical trials. The introduction of spectral-domain OCT (SDOCT) allows evaluation of retinal and subretinal structures with a potential influence on AMD development in high resolution. ${ }^{17}$ Reticular pseudodrusen (RPD), detectable as subretinal drusenoid deposits on SDOCT, are reported to be 
associated with the development of advanced AMD. ${ }^{18-23}$ Additionally, hyperreflective dots (HRD) are frequently present in eyes with intermediate $\mathrm{AMD}$ or $\mathrm{CNV}$ and are presumed to indicate imminent CNV activity or GA development. ${ }^{24-26}$

In this study, we aimed to identify possible markers for an early or late onset of the advanced stage in AMD by analyzing morphologic characteristics of the fellow eye in patients with early onset of unilateral neovascular AMD (early-onset CNV group) compared to AMD patients older than 80 years with either new-onset unilateral CNV (late-onset CNV group) or with nonneovascular AMD (no CNV group).

\section{Methods}

\section{Data Collection}

For this retrospective study, the clinical database of the Department of Ophthalmology at the University of Cologne was screened for patients older than 50 years with neovascular AMD. Clinical and imaging data of 527 consecutive patients were reviewed. For inclusion in the study, patients were required to have newly diagnosed unilateral $\mathrm{CNV}$ secondary to AMD and color fundus photographs (FP), SDOCT volume scans, and fluorescein angiography (FA) images available. Signs for AMD had to be present in at least one eye. Patients between 50 and 65 years of age meeting the inclusion criteria were included in the early-onset CNV group, and patients older than 80 years were included in the late-onset CNV group. Patients between 66 and 79 years of age were excluded from this study.

Additionally, the European Genetic Database (EUGENDA, www.eugenda.org [in the public domain]) was screened for patients with available FPs and SDOCT scans, and all patients with nonneovascular AMD and at least 80 years of age were included in the no-CNV group. Further, two age-matched comparator groups of 50 individuals each without AMD were collected from the EUGENDA database (one group 50-65 years of age and one group $>80$ years of age).

For all patients, data collection included age, sex, and ocular comorbidity. Patients with poor image quality or patients demonstrating any other ophthalmic disease that may affect grading were excluded from this study. Fundus photographs were performed using the Canon Uvi fundus camera (Tokyo, Japan) at the $40^{\circ}$ setting. Fluorescein angiography and SDOCT volume scans were performed using the Spectralis HRA and SDOCT (Heidelberg Engineering, Heidelberg, Germany). This research adhered to the tenets set forth in the Declaration of Helsinki.

\section{Image Assessment}

Images were analyzed by two senior graders (TS and LS) of the Cologne Image Reading Center (CIRCL) according to the CIRCL standard grading protocol. Age-related macular degeneration staging was performed on FP for all cases. Early AMD was defined as presence of at least 10 small drusen $(<63-\mu \mathrm{m}$ diameter) combined with pigmentary abnormalities or at least one intermediate-size drusen (63-124- $\mu$ m diameter) within the Early Treatment Diabetic Retinopathy Study (ETDRS) grid. Intermediate AMD was defined as 1 to 15 intermediate drusen or at least one large druse ( $\geq 125-\mu \mathrm{m}$ diameter). Neovascular AMD was considered present if CNV secondary to AMD was detected, and GA was defined as atrophy of the retinal pigment epithelium (RPE) larger than $>175-\mu \mathrm{m}$ diameter with involvement of the central circle of the ETDRS grid.

The fellow eyes in the early-onset CNV group and in the late-onset $\mathrm{CNV}$ group (eyes without $\mathrm{CNV}$ ) and the eyes with the higher AMD staging in the no-CNV group were used to evaluate various morphologic features on FPs, including presence of $>20$ drusen, presence of any large drusen, RPE atrophy (sharply demarcated, depigmented area $>175-\mu \mathrm{m}$ diameter with increased visibility of choroidal vessels), or pigmentary abnormalities (hypo- or hyperpigmentation other than RPE atrophy) within the ETDRS grid and presence of $>20$ extramacular drusen (EMD, defined as drusen inside field 2 but outside of the ETDRS grid). The SDOCT volume scans were evaluated for the presence of HRD and RPD.

\section{Statistical Analysis}

Statistical analysis was performed using SPSS software version 21.0 (IBM Software and Systems, Armonk, NY, USA). Binarycoded logistic regression was used to examine associations between patient groups and grading features. Odds ratios (OR) and $95 \%$ confidence intervals (CI) were computed, and $P$ values $\leq 0.05$ were considered statistically significant.

\section{Results}

\section{Patient Characteristics}

A total of 202 patients were included in our study. Fifty-seven patients were included in the early-onset CNV group (mean age, $61.41 \pm 3.77$ years; range, $50-65$ years), 47 patients in the late-onset $\mathrm{CNV}$ group (mean age, $83.31 \pm 3.43$ years; range, 80-95 years), and 98 patients in the no-CNV group (mean age, $86.81 \pm 5.13$ years; range, $80-101$ years). Eighty-seven patients (43.1\%) were male and 115 patients (56.9\%) female.

Mean age of patients in the additional two age-matched comparator groups without AMD was $62.00 \pm 2.09$ years, range, 58 to 65 (50 individuals), and $87.10 \pm 6.10$ years, range, 80 to 97 years (50 individuals).

An overview about grading parameters and AMD staging is given in Table 1.

\section{Analysis of Morphologic Parameters}

Fellow eyes in the early-onset CNV group showed significantly more often $>20$ drusen within the ETDRS grid than fellow eyes in the late-onset CNV group (OR 2.93, CI 1.31-6.58; $P=0.01$ ) or eyes in the no-CNV group (OR 2.17, CI 1.12-4.22; $P=0.02$ ). Appearance of large drusen and pigmentary abnormalities, as well as EMD, showed no significant differences between groups.

Retinal pigment epithelium atrophy was more frequently present in the groups of patients $>80$ years compared to the early-onset CNV group (for the late-onset CNV group: OR 9.43, CI 1.99-44.68; $P=0.005$; for the no-CNV group: OR 8.43, CI $1.91-37.28 ; P=0.005)$. Presence of RPD was also significantly higher in the groups of patients $>80$ years compared to the early-onset $\mathrm{CNV}$ group (for the late-onset CNV group: OR 26.86, CI 9.36-77.05; $P=9.38 \times 10^{-10}$, for the no-CNV group: OR 2.50 , CI $1.12-5.55 ; P=0.03$ ). In patients $>80$ years, RPD were more frequently present in the late-onset CNV group compared to the no-CNV group (OR 10.76, CI 4.35-26.57; $P=$ $2.63 \times 10^{-7}$ ).

Hyperreflective dots occurred more frequently in the lateonset CNV group than in the early-onset CNV group (OR 3.32, CI $1.48-7.46 ; P=0.004$ ) or the no-CNV group (OR 2.93, CI $1.42-6.10 ; P=0.004)$.

A comparison between all groups regarding the various morphologic parameters is presented in Table 2 .

Differences between the early-onset CNV group (age 50-65 years) and the late-onset $\mathrm{CNV}$ and no-CNV groups (age $>80$ years) may partially be a result of aging alone. To analyze this, we compared two age-matched groups of individuals without 
TABLE 1. AMD Staging and Morphologic Parameters of Fellow Eyes (Early-Onset CNV and Late-Onset CNV Groups) and the Eyes With the More Severe Staging (No-CNV Group)

\begin{tabular}{|c|c|c|c|c|c|c|}
\hline $\begin{array}{l}\text { AMD Staging/ } \\
\text { Morphologic Parameters }\end{array}$ & $\begin{array}{c}\text { Early-Onset } \\
\text { CNV Group, } \\
n=57 \\
50-65 \mathrm{y}\end{array}$ & $\begin{array}{c}\text { Age-Matched } \\
\text { Comparator } \\
\text { Group, } n=50 ; \\
\quad 50-65 \text { y }\end{array}$ & $\begin{array}{l}\text { Late-Onset } \\
\text { CNV Group, } \\
\begin{array}{c}n=47 \\
>80 \\
y\end{array}\end{array}$ & $\begin{array}{c}\text { No-CNV } \\
\text { Group, } \\
\begin{array}{c}n=98 \\
>80 \\
y\end{array}\end{array}$ & $\begin{array}{c}\text { Age-Matched } \\
\text { Comparator } \\
\text { Group, } n=50 ; \\
>80 \text { y }\end{array}$ & $\begin{array}{c}\text { Total for Early- } \\
\text { Onset, Late-Onset, } \\
\text { and No-CNV } \\
\text { Groups, } n=202\end{array}$ \\
\hline No AMD & $12,21.1 \%^{*}$ & $50,100 \%$ & $3,6.4 \%^{*}$ & $0,0 \%$ & $50,100 \%$ & $15,7.4 \%$ \\
\hline Early AMD & $15,26.3 \%$ & $0,0 \%$ & $13,27.7 \%$ & $26,26.5 \%$ & $0,0 \%$ & $54,26.7 \%$ \\
\hline Intermediate AMD & $30,52.6 \%$ & $0,0 \%$ & $26,55.3 \%$ & $54,55.1 \%$ & $0,0 \%$ & $110,54.5 \%$ \\
\hline Geographic atrophy & $0,0 \%$ & $0,0 \%$ & $5,10.6 \%$ & $18,18.4 \%$ & $0,0 \%$ & $23,11.4 \%$ \\
\hline$>20$ macular drusen & $33,57.9 \%$ & $0,0 \%$ & $15,31.9 \%$ & $38,38.8 \%$ & $0,0 \%$ & $86,42.6 \%$ \\
\hline Large drusen & $30,52.6 \%$ & $0,0 \%$ & $30,63.8 \%$ & $66,67.3 \%$ & $0,0 \%$ & $126,62.4 \%$ \\
\hline Pigmentary abnormalities & $23,40.4 \%$ & $1,2 \%$ & $26,55.3 \%$ & $45,45.9 \%$ & $6,12 \%$ & $94,46.5 \%$ \\
\hline RPE atrophy & $2,3.5 \%$ & $0,0 \%$ & $12,25.5 \%$ & $23,23.5 \%$ & $0,0 \%$ & $37,18.3 \%$ \\
\hline$>20$ extramacular drusen & $16,28.1 \%$ & $1,2 \%$ & $10,21.3 \%$ & $26,26.5 \%$ & $3,6 \%$ & $52,25.7 \%$ \\
\hline Hyperreflective dots & $21,36.8 \%$ & $2,4 \%$ & $31,66.0 \%$ & $39,39.8 \%$ & $5,10 \%$ & $91,45.0 \%$ \\
\hline Reticular pseudodrusen & $10,17.5 \%$ & $1,2 \%$ & $40,85.1 \%$ & $34,34.7 \%$ & $3,6 \%$ & $84,41.6 \%$ \\
\hline
\end{tabular}

* All of these patients showed signs for AMD in the other eye.

AMD. Comparison between the comparator groups revealed no statistically significant differences for all morphologic parameters (Tables 1,2$)$. There was a trend $(P=0.07)$ for pigmentary abnormalities, indicating that these may be more frequent in elderly people.

\section{Discussion}

Prospective studies have demonstrated that large drusen and pigmentary abnormalities are associated with an increased risk for the development of $\mathrm{CNV}$ within 5 years. ${ }^{27,28}$ Additional morphologic parameters that have been reported to be associated with advanced AMD include RPD and HRD. ${ }^{18-20,22-26}$ Risk factors for CNV in the second eye include drusen number and area as well as pigmentary abnormalities within the macula. ${ }^{12}$ Early onset of the advanced stage of AMD with development of CNV results in impairment of vision over possibly many years and therefore severely impacts quality of life. Epidemiologic studies revealed that past or current smokers, homozygous carriers of the $\mathrm{Y} 402 \mathrm{H}$ risk variant in the ARMS2 gene, and patients carrying four risk alleles in $C F H$ and $A R M S 2$ have a higher risk to show a more aggressive form of the disease with development of CNV early in life. 9 However, morphologic characteristics for patients with early onset of CNV in AMD have not yet been fully understood.

In our study, $>20$ macular drusen appeared to be more frequently present in fellow eyes of patients with early onset of unilateral neovascular AMD and thus may represent an indicator for a more aggressive course of the disease. Patients $>80$ years of age showed less frequently a large number of macular drusen. There were no differences regarding drusen number between AMD patients $>80$ years with and without $\mathrm{CNV}$, suggesting that the number of drusen may have a lower relevance for CNV occurrence in elderly patients. Silva et al. ${ }^{29}$ did not find a predictive value of drusen number or area, presence of large drusen, or pigmentary changes for conversion to wet AMD during a 3-year study follow-up. ${ }^{29}$ Fridberg et al. ${ }^{30}$ reported that drusen area, as well as the presence of large drusen or hyperpigmentation, is not a consistent risk factors for an eye's development of CNV. ${ }^{30} \mathrm{He}$ proposed that whether or not these features present relevant risk factors for $\mathrm{CNV}$ development may depend on the location of the feature in relation to the fovea. According to our results, we additionally propose that the age of the patient may be another possibly influencing factor.
In our study group we found no differences between groups regarding the appearance of large drusen or pigmentary abnormalities, indicating that these parameters do not represent characteristic markers for an early or late onset of CNV. Our study did not include analyses of follow-up images, and thus was not designed to draw conclusions about the predictive value of parameters. Therefore, large drusen or pigmentary abnormalities may precede CNV development as reported in the Age-Related Eye Disease Study; however, in our study we did not detect differences between younger and older patient groups.

Macular drusen have been reported to be associated with peripheral drusen (Ersoy L, Schick T, De Graft D, et al., manuscript submitted, 2015). ${ }^{31}$ However, the relevance of EMD for the development of AMD or CNV is not yet fully understood. Previous studies indicated that EMD are not associated with known AMD risk alleles (Ersoy L, Schick T, De Graft D, et al., manuscript submitted, 2015), and thus patients with drusen exclusively outside of the ETDRS grid may have genetically the same risk to develop AMD compared to patients without any drusen. In our study, we could not identify any differences between groups regarding the occurrence of drusen outside of the ETRDS grid, indicating that EMD do not represent a characteristic marker of an early or late onset of the neovascular stage in AMD.

Retinal pigment epithelium atrophy as well as RPD appeared to be more frequently present in AMD patients over 80 years compared to patients with early onset of neovascular AMD. Retinal pigment epithelium atrophy was more frequently present in elderly patients, independently of whether they showed CNV in one eye or not. Reticular pseudodrusen are subretinal drusenoid deposits, accumulated above the RPE in the subretinal space. ${ }^{22,23}$ In our study, RPD were more frequently present in both elderly groups compared to the early-onset $\mathrm{CNV}$ group, which supports the thesis that the risk for RPD increases with age. ${ }^{32}$ Reticular pseudodrusen were most strongly associated with the late-onset CNV group compared to the no-CNV group, which is in agreement with other studies indicating that RPD are associated with neovascular AMD. ${ }^{20-22}$

Hyperreflective dots on SDOCT scans may correlate to various features including hard exudates, hemorrhage, RPE migration, or inflammatory cells. ${ }^{33}$ Schuman et al. ${ }^{34}$ studied the association with drusen, supposing that HRD could be due to the migration of macrophages that have gulped degenerative material from damaged photoreceptors. ${ }^{34}$ Hyperreflective dots 
are reported to precede $\mathrm{CNV}$ development and to be associated with CNV. ${ }^{25,35}$ This is in accordance with our results, as HRD were more frequently detected in the late-onset $\mathrm{CNV}$ group compared to the no-CNV group. This may be explained by increased inflammatory cell migration processes in patients with neovascular AMD. Hyperreflective dots additionally occurred more often in the late-onset $\mathrm{CNV}$ group than in the early-onset CNV group.

Our study is not without limitations, including its retrospective design and the relatively small number of patients per group. Further, it cannot be ruled out that differences between younger and older patient groups in our study may be a result of aging rather than a result of early or late CNV development. Comparison between age-matched comparator groups revealed no differences regarding the presence of examined morphologic parameters for individuals without AMD. In AMD patients, however, drusen may regress over time, eventually contributing to the development of RPE disturbance or atrophy. ${ }^{36-38}$

However, our study also has several strengths. All images were graded by trained reading center graders according to a standard grading protocol. The utilization of high-resolution SDOCT images allows a detailed interpretation of the complex morphologic changes occurring in neovascular AMD, including assessment of HRD, RPD, and CNV morphology.

In summary, a large number of macular drusen in the fellow eye appeared to be characteristic in patients with early onset of the neovascular stage in AMD, whereas HRD and RPD appeared to be characteristic for elderly patients with neovascular AMD. Retinal pigment epithelium atrophy has been more frequently detected in older AMD patients regardless of whether or not $\mathrm{CNV}$ was present. Prospective studies with a large number of patients converting to neovascular AMD are required to further identify morphologic characteristics of patients with a more aggressive form of the disease with early onset of $\mathrm{CNV}$.

\section{Acknowledgments}

Supported in part by the Ilse Palm Foundation, Essen, Germany.

Disclosure: T. Schick, None; L. Ersoy, None; C.B. Hoyng, None; B. Kirchhof, None; S. Liakopoulos, Heidelberg Engineering (R)

\section{References}

1. Bressler NM, Doan QV, Varma R, et al. Estimated cases of legal blindness and visual impairment avoided using ranibizumab for choroidal neovascularization: non-Hispanic white population in the United States with age-related macular degeneration. Arch Ophthalmol. 2011;129:709-717.

2. Smith W, Assink J, Klein R, et al. Risk factors for age-related macular degeneration: pooled findings from three continents. Ophthalmology. 2001;108:697-704.

3. Friedman DS, O'Colmain BJ, Munoz B, et al. Prevalence of agerelated macular degeneration in the United States. Arch Ophthalmol. 2004;122:564-572.

4. Klein R, Klein BE, Knudtson MD, Meuer SM, Swift M, Gangnon RE. Fifteen-year cumulative incidence of age-related macular degeneration: the Beaver Dam Eye Study. Ophthalmology. 2007; 114:253-262.

5. Rosenfeld PJ, Brown DM, Heier JS, et al. Ranibizumab for neovascular age-related macular degeneration. $N$ Engl J Med. 2006;355:1419-1431.

6. Fritsche LG, Chen W, Schu M, et al. Seven new loci associated with age-related macular degeneration. Nat Genet. 2013;45: 433-439, 439e1-e2. 
7. Klein ML, Francis PJ, Ferris FL III, Hamon SC, Clemons TE. Risk assessment model for development of advanced age-related macular degeneration. Arch Ophthalmol. 2011;129:15431550 .

8. Ferris FL, Davis MD, Clemons TE, et al. A simplified severity scale for age-related macular degeneration: AREDS Report No. 18. Arch Ophthalmol. 2005;123:1570-1574.

9. Lechanteur YT, van de Camp PL, Smailhodzic D, et al. Association of smoking and CFH and ARMS2 risk variants with younger age at onset of neovascular age-related macular degeneration. JAMA Ophthalmol. 2015;133:533-541.

10. Gangnon RE, Lee KE, Klein BE, Iyengar SK, Sivakumaran TA, Klein R. Severity of age-related macular degeneration in 1 eye and the incidence and progression of age-related macular degeneration in the fellow eye: the Beaver Dam eye study. JAMA Opbthalmol. 2015;133:125-132.

11. Five-year follow-up of fellow eyes of patients with age-related macular degeneration and unilateral extrafoveal choroidal neovascularization. Macular Photocoagulation Study Group. Arch Ophthalmol. 1993;111:1189-1199.

12. Risk factors for choroidal neovascularization in the second eye of patients with juxtafoveal or subfoveal choroidal neovascularization secondary to age-related macular degeneration. Macular Photocoagulation Study Group. Arch Ophthalmol. 1997;115:741-747.

13. Gregor Z, Bird AC, Chisholm IH. Senile disciform macular degeneration in the second eye. Br J Ophthalmol. 1977;61: 141-147.

14. Pieramici DJ, Bressler SB. Age-related macular degeneration and risk factors for the development of choroidal neovascularization in the fellow eye. Curr Opin Opbthalmol. 1998;9: 38-46.

15. Roy M, Kaiser-Kupfer M. Second eye involvement in agerelated macular degeneration: a four-year prospective study. Eye (Lond). 1990;4(pt 6):813-818.

16. Huang D, Swanson EA, Lin CP, et al. Optical coherence tomography. Science. 1991;254:1178-1181.

17. Keane PA, Patel PJ, Liakopoulos S, Heussen FM, Sadda SR, Tufail A. Evaluation of age-related macular degeneration with optical coherence tomography. Surv Ophthalmol. 2012;57: 389-414.

18. Schmitz-Valckenberg S, Steinberg JS, Fleckenstein M, Visvalingam S, Brinkmann CK, Holz FG. Combined confocal scanning laser ophthalmoscopy and spectral-domain optical coherence tomography imaging of reticular drusen associated with agerelated macular degeneration. Ophthalmology. 2010;117: 1169-1176.

19. Finger RP, Wu Z, Luu CD, et al. Reticular pseudodrusen: a risk factor for geographic atrophy in fellow eyes of individuals with unilateral choroidal neovascularization. Ophthalmology. 2014; 121:1252-1256.

20. Pumariega NM, Smith RT, Sohrab MA, Letien V, Souied EH. A prospective study of reticular macular disease. Ophthalmology. 2011;118:1619-1625.

21. Arnold JJ, Sarks SH, Killingsworth MC, Sarks JP. Reticular pseudodrusen. A risk factor in age-related maculopathy. Retina. 1995;15:183-191.

22. Zweifel SA, Imamura Y, Spaide TC, Fujiwara T, Spaide RF. Prevalence and significance of subretinal drusenoid deposits (reticular pseudodrusen) in age-related macular degeneration. Ophthalmology. 2010;117:1775-1781.
23. Zweifel SA, Spaide RF, Curcio CA, Malek G, Imamura Y. Reticular pseudodrusen are subretinal drusenoid deposits. Ophthalmology. 2010;117:303-312, e301.

24. Christenbury JG, Folgar FA, O'Connell RV, et al. Progression of intermediate age-related macular degeneration with proliferation and inner retinal migration of hyperreflective foci. Ophthalmology. 2013;120:1038-1045.

25. Coscas G, De Benedetto U, Coscas F, et al. Hyperreflective dots: a new spectral-domain optical coherence tomography entity for follow-up and prognosis in exudative age-related macular degeneration. Ophthalmologica. 2013;229:32-37.

26. Ouyang Y, Heussen FM, Hariri A, Keane PA, Sadda SR. Optical coherence tomography-based observation of the natural history of drusenoid lesion in eyes with dry age-related macular degeneration. Ophthalmology. 2013;120:2656-2665.

27. Chew EY, Clemons TE, Agron E, et al. Ten-year follow-up of age-related macular degeneration in the age-related eye disease study: AREDS report no. 36. JAMA Ophthalmol. 2014;132: 272-277.

28. Davis MD, Gangnon RE, Lee LY, et al. The Age-Related Eye Disease Study severity scale for age-related macular degeneration: AREDS Report No. 17. Arch Ophthalmol. 2005;123: $1484-1498$.

29. Silva R, Cachulo ML, Fonseca P, et al. Age-related macular degeneration and risk factors for the development of choroidal neovascularisation in the fellow eye: a 3-year follow-up study. Ophthalmologica. 2011;226:110-118.

30. Friberg TR, Bilonick RA, Brennen P. Is drusen area really so important? An assessment of risk of conversion to neovascular AMD based on computerized measurements of drusen. Invest Ophthalmol Vis Sci. 2012;53:1742-1751.

31. Munch IC, Ek J, Kessel L, et al. Small, hard macular drusen and peripheral drusen: associations with AMD genotypes in the Inter99 Eye Study. Invest Ophthalmol Vis Sci. 2010;51:23172321.

32. Boddu S, Lee MD, Marsiglia M, Marmor M, Freund KB, Smith RT. Risk factors associated with reticular pseudodrusen versus large soft drusen. Am J Ophthalmol. 2014;157:985-993.

33. Bolz M, Schmidt-Erfurth U, Deak G, et al. Optical coherence tomographic hyperreflective foci: a morphologic sign of lipid extravasation in diabetic macular edema. Ophthalmology. 2009;116:914-920.

34. Schuman SG, Koreishi AF, Farsiu S, Jung SH, Izatt JA, Toth CA. Photoreceptor layer thinning over drusen in eyes with agerelated macular degeneration imaged in vivo with spectraldomain optical coherence tomography. Ophthalmology. 2009; 116:488-496, e482.

35. Giani A, Luiselli C, Esmaili DD, et al. Spectral-domain optical coherence tomography as an indicator of fluorescein angiography leakage from choroidal neovascularization. Invest Ophthalmol Vis Sci. 2011;52:5579-5586.

36. Bressler NM, Munoz B, Maguire MG, et al. Five-year incidence and disappearance of drusen and retinal pigment epithelial abnormalities. Waterman study. Arch Ophthalmol. 1995;113: 301-308.

37. Suzuki M, Curcio CA, Mullins RF, Spaide RF. REFRACTILE DRUSEN: clinical imaging and candidate histology. Retina. 2015;35:859-865.

38. Toy BC, Krishnadev N, Indaram M, et al. Drusen regression is associated with local changes in fundus autofluorescence in intermediate age-related macular degeneration. Am J Opbthalmol. 2013;156:532-542, e531. 